\title{
Consistent two-dimensional chiral gravity
}

\author{
A.Smailagic', \\ International Center for Theoretical Physics, \\ Miramare, 34014-Trieste, Italy, \\ and, \\ Dipartimento di Fisica Teorica dell'Università, \\ Strada Costiera 11, 34014-Trieste, Italy, \\ E.Spalluccið \\ Dipartimento di Fisica Teorica dell'Università, \\ Strada Costiera 11, 34014-Trieste, Italy, \\ Istituto Nazionale di Fisica Nucleare, Sezione di Trieste, \\ Strada Costiera 11, 34014-Trieste, Italy,
}

\begin{abstract}
We study chiral induced gravity in the light-cone gauge and show that the theory is consistent for a particular choice of chiralities. The corresponding Kac-Moody central charge has no forbidden region of complex values. Generalized analysis of the critical exponents is given and their relation to the $S L(2, R)$ vacuum states is elucidated. All the parameters containing information about the theory can be traced back to the characteristics of the group of residual symmetry in the light-cone gauge.
\end{abstract}

\footnotetext{
${ }^{1}$ E-mail address: anais@ictp.trieste.infn.it

${ }^{2}$ E-mail address: spallucci@vstst0.ts.infn.it
} 


\section{Introduction}

Two-dimensional induced gravity has become an interesting field of investigations since the early days of Polyakov functional integral formulation of string theory [1]. Such a description enables a thorough investigation of the string dynamics only at the critical dimension, where there is no need to worry about gravitational quantum effects. On the other hand, the occurrence of the anomaly in off-critical string models makes unavoidable the presence of gravitational quantum effects, and introduces a further technical problem in actual calculations. A "proper" gauge choice becomes an essential step in the formal developments of computations. Conventionally, off-critical models have been dealt with in the conformal gauge [2]. The advantage of choosing such a gauge is to give the effective action a local form, i.e. the effective dynamics of the Weyl degree of freedom is described by Liouville local field theory. However, there is a double price to pay: first, quantization becomes non-trivial due to the dependence on the Weyl factor; second, one is faced with the, so far unresolved, problem [3] to introduce, at the perturbative level, a suitable regularization procedure. Therefore, the choice of the conformal gauge exchanges non-locality with all the quantization problems of the Liouville field theory [4]. Recently, some progress has been made in the quantization of Liouville theory, as well as in treating the strong coupling regime in the conformal gauge [5], [6]. Under this respect, it is somehow amazing that the light-cone gauge choice is so "clever" to circumvent these difficulties [7] ! The regularization problem does not exist. The effective action, though apparently in non-local form, can be handled and the model is completely solvable. Furthermore, an unexpected residual $S L(2, R)$ symmetry shows up. Due to the solvability of the model, the Kac-Moody central charge can be renormalized in closed form and various critical exponents can be evaluated. Then, it is possible to compare them with the corresponding quantities in statistical physics [8], and verify a complete agreement among certain non-critical string theories and some definite

statistical models. However, the allowed range of values for the central charge suffers from a gap corresponding to complex, unphysical, values and forbids the extrapolation of low dimensional results, corresponding to various statistical models, to higher dimensional ones relevant to string models. This gap can be narrowed down by exploiting $N=1$ supersymmetry and even avoided in the $N=2$ supersymmetric model [9]. 
The motivation of the present paper is drawn from the known fact that chiral induced gauge theories [10], though anomalous, can be consistent under certain conditions. With the same hope, we shall investigate the light-cone gauge structure of the chiral induced gravity. This choice is motivated by the presence of a Lorentz anomaly which cannot be removed by any regularization procedure [11]. As a result, the Lorentz mode shows up in the spectrum as a physical degree of freedom associated with an arbitrary parameter interpolating over different regularization schemes.

One of the objectives of this paper is to employ the freedom introduced by the regularization parameter with the hope to eliminate the region of complex values of the central charge, as an alternative way to the use of $N=2$ supersymmetry in non chiral models.

Another goal is to investigate critical exponents within light-cone gauge description, and connect them to the characteristics of the residual symmetry group.

Finally, we would like to establish complete correspondence among the parameters characterizing induced gravity models in the light-cone and conformal gauges. While carrying on this program, we have found intriguing possibility of a generalization of various formulae, that could lead to a simple description of all the known chiral and non-chiral gravity models, as well as their supersymmetric versions. Instead of bothering with a complex and different structures of various models, and other technical details, we hope to absorb all the essential characteristics of the mentioned models within $a$ couple of general parameters from which one can work out the details of any desired model. This, certainly, would be an interesting simplification in handling the large number of specific results throughout the literature.

The paper is planned as follows.

In Sect. 2 we discuss the $N=0$ chiral gravity model, and its classical symmetries before any gauge choice. The purpose of this section is to offer some technical details and stress the differences between chiral and non chiral models. Since we are using a symmetric version of the induced action we recall the connection to the asymmetric form of the same quantity, more often appearing in the literature. This model is more involved than the non chiral one, due to the explicit presence of the spin connection. We describe conservation laws and the corresponding symmetries assuming the zweibein as the basic variable in the model. Our approach leads to an easy comparison of the components of the energy-momentum tensor in different gauges. 
In Sect.3 we give a detailed description of physical quantities in the lightcone gauge, and discuss the residual symmetries. We show that some of the components of the energy-momentum tensor are generators of these residual symmetries, and can be described in the Sugawara form. The remaining components produce equations of motion for the dynamical components of the zweibein. Then, we study the general properties of the roots of the quadratic equations that describe the renormalized $S L(2, R)$ Kac-Moody central charge. We also analyze the definition of the renormalized gravitational scaling dimension and the string susceptibility coefficient in terms of the scaling weights of the $S L(2, R)$ currents. In this way, we obtain a very simple meaning of these quantities and the relations to scale operators connecting various physical states. We also obtain a generalized form of the KPZ equation.

In Sect. 4 we investigate the connection between the characteristic parameters of the conformal and light-cone gauge and establish a number of interesting general relations.

In Sect.5 we analyze various restrictions on the number of matter fields and a free parameter characterizing the chiral model, resulting from the requirement of reality of the parameters characterizing conformal and light-cone gauge. At this point essential difference between chiral and non-chiral model is pointed and discussed.

Finally, Sect.5 is devoted to a discussion of our results. We list a number of improvements and new results in chiral models.

\section{General description of the chiral model}

Supersymmetric and non-supersymmetric versions of the non-chiral models have been studied extensively and details can be found in the literature [7], [9], [12]. Details of chiral models are less known and we shall give a comprehensive description of the structure of their non-supersymmetric version. In this way we avoid complications due to the superspace description [13], while maintaining all the important characteristics necessary for our discussion.

The model is described by the induced action resulting from the integration of matter fields [14] 


$$
\begin{aligned}
S_{\text {symm. }} & =\frac{1}{2} \int d^{2} x \sqrt{-g}\left[\mathcal{R} \frac{1}{\nabla^{2}} \mathcal{R}+b \omega^{2}\right] \\
& =\frac{1}{2} \int d^{2} x \sqrt{-g}\left[\nabla^{\mu} \phi \nabla_{\mu} \phi+2 \mathcal{R} \phi+b \omega^{2}\right]
\end{aligned}
$$

where the convenient notation has been adopted

$$
\mathcal{R}=\alpha R+\beta \nabla_{\mu} \omega^{\mu}, \quad \nabla^{2} \phi=\mathcal{R},
$$

in order to put action (2.1) in the compact, and local, form (2.2). $\nabla_{\mu}$ is the covariant derivative with respect to the Christoffel symbol, and $\omega_{\mu}=\epsilon_{a b} e^{b \nu} \nabla_{\mu} e^{a}{ }_{\nu}$ is a Lorentz connection. We adopt a symmetric version of the induced action because it is easier to work with, but it can be quickly translated into the asymmetric form usually appearing in the literature [15]

$$
S_{\text {asymm. }}=\frac{1}{192 \pi} \int d^{2} x \sqrt{-g}\left[R \frac{1}{\nabla^{2}}\left(\mathcal{N} R+\Delta \mathcal{N} \nabla_{\mu} \omega^{\mu}\right)+\frac{1}{2} \widehat{b} \omega^{2}\right]
$$

through the following identification of appropriate parameters

$$
\begin{aligned}
\alpha^{2}+\beta^{2} & =\frac{\mathcal{N}}{96 \pi}, \\
\alpha \beta & =\frac{\Delta \mathcal{N}}{192 \pi}, \\
\widehat{b} & =192 \pi\left(b-\beta^{2}\right), \\
\mathcal{N} & =n_{+}+n_{-}, \\
\Delta \mathcal{N} & =n_{-}-n_{+},
\end{aligned}
$$

where, $n_{+}, n_{-}$are the numbers of left and right chiral fermions ( a priori $n_{+} \neq$ $n_{-}$) whose quantum fluctuations induce the chiral gravitational action. The comparison of the actions (2.1),(2.4) is possible because the corresponding non-local parts differ only by a local $\omega^{2}$ term, which can be absorbed in a local counter-term through the redefinition of the free regularization parameter $b$. The non chiral model corresponds to the choice $n_{+}=n_{-}$, or $\beta=0$, and $b=0$.

The energy-momentum tensor for this model is given by 


$$
\begin{aligned}
T_{\mu \nu} \equiv & -\frac{1}{\operatorname{det} \mathbf{e}} e_{a \mu} \frac{\delta S}{\delta e_{a}{ }^{\nu}} \\
= & 2 \alpha \nabla_{\mu} \nabla_{\nu} \phi-\nabla_{\mu} \phi \nabla_{\nu} \phi+\beta\left(\omega_{\mu} \nabla_{\nu} \phi+\omega_{\nu} \nabla_{\mu} \phi\right) \\
& -b \omega_{\mu} \omega_{\nu}-e^{c}{ }_{\mu} e_{c \nu}\left(2 \alpha \nabla^{2} \phi-\frac{1}{2} \nabla^{\rho} \phi \nabla_{\rho} \phi+\beta \omega^{\rho} \nabla_{\rho} \phi-\frac{1}{2} b \omega^{2}\right) \\
& -2 \beta\left[\epsilon_{\mu \nu} \nabla^{2} \phi+\epsilon_{\nu \rho} \nabla^{\rho} \nabla_{\mu} \phi\right]+2 b\left[\epsilon_{\mu \nu} \nabla \omega+\epsilon_{\nu \rho} \nabla^{\rho} \omega_{\mu}\right] .
\end{aligned}
$$

It is worth reminding that the energy momentum tensor defined with respect to the zweibein is not a priori symmetric in the indices. This fact will be visible through the presence of the Lorentz anomaly [. The conservation laws satisfied by $T_{\mu \nu}$ are

$$
\begin{aligned}
\nabla^{\mu} T_{\mu \nu} & =0, \\
T & =2\left(b+\alpha^{2}\right) R+2 \alpha \beta \nabla \omega, \\
\epsilon^{\mu \nu} T_{\mu \nu} & =-2 \alpha \beta R+2\left(b-\beta^{2}\right) \nabla \omega,
\end{aligned}
$$

Equations (2.7) show that we have adopted approach of maintaining maximal possible residual symmetry after integration of the matter fields, which produces the anomalous action (2.2). Also, we have chosen to maintain diffeomorfism invariance at the expenses of the Lorentz and Weyl symmetry. Lorentz anomaly is a genuine chiral effect, and it can be restored only in the non-chiral case $(\beta=0)$ by the choice $b=0$. It is worth mentioning that the equations (2.7) follow directly from equation (2.6) and are, therefore, off-shell anomalies. The essence of the approach described in our paper is to consider induced anomalous action (2.1) as the "classical" action for 2D gravity. In other words, anomalies are crucial to render certain components of the metric (or zweibein) dynamical, and the anomaly equations are the equations of motion for those dynamical components.

Since diffeomorfism invariance is still a gauge symmetry of the effective action (2.1), we can use this gauge freedom to dispose off some of the components of $e_{\mu}^{a}$. So, one is faced with the choice of gauge. We shall study the model in the light-cone gauge, where we can exploit the Kac-Moody structure of (chiral) gravity. Moreover, we shall establish detailed relationships

\footnotetext{
${ }^{3}$ The non-chiral version of $(\sqrt{2.6})$ has already been given in 16
} 
with the parameters characterizing the conformal gauge. In order for the reader to be able to follow our formulae, we give the decomposition

$$
\begin{aligned}
\omega_{\mu} & =\partial_{\mu} L-\epsilon_{\mu \nu} \partial^{\nu} \varphi+\bar{\omega}_{\mu} \\
\bar{\omega}_{\mu} & =\epsilon_{a b} \bar{e}^{b \nu} \nabla_{\mu} \bar{e}^{a}{ }_{\nu} \\
e^{a \nu} & =e^{\varphi / 2}\left(\begin{array}{cc}
\cosh L / 2 & -\sinh L / 2 \\
-\sinh L / 2 & \cosh L / 2
\end{array}\right) \bar{e}^{a \nu}
\end{aligned}
$$

where, $L$ is the Lorentz degree of freedom, $\varphi$ is the Weyl degree of freedom, and the rest of the components is contained in barred quantities. So, one can easily switch between the two gauges.

\section{Light-cone gauge}

\subsection{Residual symmetries of the chiral model}

To introduce the problem, we shall recall some basic results obtained in the light-cone gauge [17]. The metric element is given by

$$
d s^{2}=d x^{+} d x^{-}+h_{++} d x^{+} d x^{+},
$$

and its form is preserved by the following residual symmetry transformation

$$
\begin{aligned}
\delta x^{+} & =\epsilon^{+}\left(x^{+}\right), \\
\delta x^{-} & \equiv \epsilon^{-}\left(x^{+}, x^{-}\right)=\eta^{-}\left(x^{+}\right)-x^{-} \partial_{+} \epsilon^{+}\left(x^{+}\right)
\end{aligned}
$$

At this point we would like to stress that diffeomorfisms are the only remaining symmetry of the induced action due to the presence of Lorentz and Weyl anomaly (2.2). Therefore, the later two symmetries are no more an issue, and we shall not be using them. Diffeomorfisms will be used to get rid of some of the gauge degrees of freedom leading to the above choice of the line element.

In this case residual diffeomorfisms reduce to the conformal transformation in the left-sector, while in the right-sector they represent a more complex transformation, as written in (3.2). 
The Lorentz degree of freedom $L$, and the metric component $h_{++}$can be defined through the zweibein components given by (2.8) as

$$
\begin{aligned}
e^{L} & =e^{\widehat{+}}+e^{-} \widehat{-}, \\
h_{++} & =e^{\widehat{-}}+e^{\widehat{+}} .
\end{aligned}
$$

while components of the spin connection and of the Christoffel symbols, are

$$
\begin{aligned}
\omega_{+} & =\partial_{+} L+2 \partial_{-} h_{++}, \\
\omega_{-} & =\partial_{-} L \\
\Gamma^{-}{ }_{+-} & =-\Gamma^{+}+\partial_{++} h_{++}, \\
\Gamma^{-}{ }_{++} & =\partial_{+} h_{++}+2 h_{++} \partial_{-} h_{++} .
\end{aligned}
$$

Lorentz indices in equation (3.3) and equation (2.8) (Latin) will be denoted by a hat when written in terms of light-cone components to distinguish them from ( un-hatted ) world (Greek) indices. However, hatted indices will not further appear in our formulae. The dynamical components of the metric and the zweibein vary under the transformation (3.2) as

$$
\begin{aligned}
\delta h_{++} & =\epsilon^{+} \partial_{+} h_{++}+2 h_{++} \partial_{+} \epsilon^{+} \\
& -x^{-} \partial_{-} h_{++} \partial_{+} \epsilon^{+}-x^{-} \partial_{+}^{2} \epsilon^{+}+\eta^{-} \partial_{-} h_{++}+\partial_{+} \eta^{-} \\
\delta L & =\epsilon^{+} \partial_{+} L-x^{-} \partial_{+} \epsilon^{+} \partial_{-} L+\partial_{+} \epsilon^{+}+\eta^{-} \partial_{-} L .
\end{aligned}
$$

We would like to find the generators of the residual symmetries (3.2) which should be appropriate combination of the components of energy momentum tensor such that it is dependent only on the coordinate $x^{+}$. In order to find such a combination one can start from the symmetry condition (2.7) which in this gauge can be written as

$$
\partial_{-}\left(T_{++}-2 h_{++} T_{+-}\right)+\partial_{+} T_{+-}=0
$$

The above equation has a solution

$$
T_{++}\left(x^{+}, x^{-}\right)=\widetilde{T}_{++}\left(x^{+}\right)+2 h_{++}\left(x^{+}, x^{-}\right) T_{+-}\left(x^{+}\right)-x^{-} \partial_{+} T_{+-}\left(x^{+}\right)
$$


$T_{++}\left(x^{+}, x^{-}\right)$and $T_{+-}\left(x^{+}\right)$are components of energy momentum tensor given by (2.6), and $\widetilde{T}_{++}$is the generator of the residual conformal transformation in the left-sector. Its explicit form will be given later. We shall also show that the equation of motion for the induced gravity restrict $T_{+-}$to be function of $x^{+}$alone. The components of energy momentum tensor coupling to the dynamical degrees of freedom $h_{++}, L$ are

$$
\begin{aligned}
T_{--}(\phi) & =\left[2(\alpha+\beta) \partial_{-}^{2} \phi-\left(\partial_{-} \phi\right)^{2}+2 \beta \omega_{-} \partial_{-} \phi-2 b \partial_{-} \omega_{-}-b \omega_{-}^{2}\right] \\
\epsilon^{\mu \nu} T_{\mu \nu} & =-2 \alpha \beta R+2\left(b-\beta^{2}\right) \nabla \omega .
\end{aligned}
$$

and they lead to the equations of motion given by

$$
\begin{aligned}
& {\left[(\alpha \pm \beta)^{2}+\left(b-\beta^{2}\right)\left(1 \mp \frac{\alpha \beta}{b-\beta^{2}}\right)^{2}\right] \partial_{-}^{3} h_{++}+\alpha \beta \partial_{-}^{2} A_{+}=0,} \\
& \left(b-\beta^{2}\right) \partial_{-} A_{+}=0, \\
& \left(b-\beta^{2}\right) \neq 0 .
\end{aligned}
$$

In order to write equations (3.9) in the simple looking form we have introduced the following redefinitions

$$
\begin{aligned}
A_{+} & =D_{+} L+\left(1-\frac{\alpha \beta}{b-\beta^{2}}\right) \partial_{-} h_{++}, \\
D_{+} L & =\partial_{+} L-h_{++} \partial_{-} L .
\end{aligned}
$$

We would like to mention that the consistency of the equations of motion (3.9), and redefinitions (3.10) require $b \neq \beta^{2}$ and, therefore, the previous limit leading to the non-chiral model $(\beta=0, b=0)$ cannot be applied anymore. Of course, one could have continue to work with the original Lorentz field $L$, but the fields $h_{++}, L$ would not decouple, leading to off-diagonal propagators and less elegant looking expressions. The non-chiral results are now simply obtained by dropping all the contribution of the $A_{+}$field.

The components of the energy momentum tensor following from the equation (2.6), and coupling to the gauge degrees of freedom $h_{--}, \varphi$ are

$$
T_{+-}=2 Q \partial_{-}^{2} h_{++},
$$




$$
\begin{aligned}
T_{++}-2 h_{++} T_{+-} & =\left[-\left(\partial_{-} h_{++}\right)^{2}+2 h_{++} \partial_{-}^{2} h_{++}-2 Q \partial_{-} \partial_{+} h_{++}\right] \\
& +\left[-A_{+}^{2}+2 Q_{\text {Lorentz }} \partial_{+} A_{+}\right]
\end{aligned}
$$

where, we have rescaled fields as $h_{++} \rightarrow\left(1 / Q_{l c}\right) h_{++}$f , and $A_{+} \rightarrow$ $\left(1 / \sqrt{b-\beta^{2}}\right) A_{+}$. The constants $Q_{\text {lc }}, Q_{\text {Lorentz }}$ are defined as

$$
\begin{aligned}
Q_{l c} & \equiv\left[-(\alpha \pm \beta)^{2}-\left(b-\beta^{2}\right)\left[1 \mp \frac{\alpha \beta}{b-\beta^{2}}\right]^{2}\right]^{1 / 2}, \\
Q_{\text {Lorentz }} & \equiv\left(b-\beta^{2}\right)^{1 / 2}\left(1+\frac{\alpha \beta}{b-\beta^{2}}\right)
\end{aligned}
$$

and we made use of the identity

$$
b\left(1+\frac{\alpha^{2}}{b-\beta^{2}}\right) \equiv(\alpha \pm \beta)^{2}+\left(b-\beta^{2}\right)\left[1 \mp \frac{\alpha \beta}{b-\beta^{2}}\right]^{2}
$$

Solutions of the equations of motion (3.9) can be written in terms of four functions $J_{i}\left(x^{+}\right), \bar{J}_{+}\left(x^{+}\right)$

$$
\begin{aligned}
h_{++}(x) & =J_{++}\left(x^{+}\right)-2 x^{-} J_{+}\left(x^{+}\right)+\left(x^{-}\right)^{2} J_{0}\left(x^{+}\right), \\
A_{+}(x) & =\bar{J}_{+}\left(x^{+}\right) .
\end{aligned}
$$

With the help of these solutions one can write the generators of the residual symmetries as

$$
\begin{aligned}
& T_{+-}=J_{0}\left(x^{+}\right), \\
& T_{++}=\frac{1}{K} \eta^{a b} J_{a} J_{b}+\partial_{+} J_{+}+\frac{1}{K_{U(1)}} \bar{J}_{+}^{2}+\partial_{+} \bar{J}_{+} .
\end{aligned}
$$

\footnotetext{
${ }^{4}$ Since we are considering two different gauge choices, we shall denote by a subscript, "lc" for light-cone, and "conf" for conformal gauge, all the quantities that a priori gauge dependent and therefore different. It may, however, turn out a posteriori that some of these quantities are actually equal. In that case we shall drop the appropriate subscript.
} 
We have further rescaled functions $J_{a}$ as $J_{a} \rightarrow\left(1 / 4 Q_{l c}\right) J_{a}$ and $\bar{J}_{+}$as $\bar{J}_{+} \rightarrow$ $\left(1 / 2 Q_{\text {Lorentz }}\right) \bar{J}_{+}$

From the above equation it is visible that the generators are functions of the coordinate $x^{+}$only. The functions $J_{a}, \bar{J}_{+}$satisfy the Dirac bracket structure

$$
\begin{aligned}
\left\{J^{a}\left(x^{+}\right), J^{b}\left(x^{+}\right)\right\} & =-\frac{K}{2} \frac{\eta^{a b}}{\left(x^{+}-y^{+}\right)^{2}}+\frac{f^{a b}{ }_{c} J^{c}\left(y^{+}\right)}{\left(x^{+}-y^{+}\right)}+\text {reg. } \\
\left\{\bar{J}\left(x^{+}\right), \bar{J}\left(x^{+}\right)\right\} & =-\frac{K_{U(1)}}{2} \frac{1}{\left(x^{+}-y^{+}\right)^{2}}+\text { reg. } \\
\left\{J^{a}\left(x^{+}\right), \bar{J}\left(x^{+}\right)\right\} & =\text {reg. }
\end{aligned}
$$

and $K, K_{U(1)}$ are given by

$$
\begin{aligned}
K & =-4 Q_{l c}^{2}, \\
K_{U(1)} & =-4 Q_{\text {Lorentz }}^{2} .
\end{aligned}
$$

The above results show that chiral gravity has an underlying $S L(2, R) \otimes U(1)$ Kac-Moody structure: $f^{a b}{ }_{c}$ and $\eta^{a b}$ being the structure constants and the metric of the $S L(2, R)$ current algebraf. The explicit form of the $S L(2, R) \otimes$ $U(1)$ generators is

$$
\begin{aligned}
l_{i}^{+} & =\left(x_{i}^{-}\right)^{2} \partial_{-i}+2\left(\lambda+\frac{\alpha \beta}{a^{\prime}-\beta^{2}} q\right) x_{-i}, \\
l_{i}^{0} & =x_{i}^{-} \partial_{-i}+\left(\lambda+\frac{\alpha \beta}{a^{\prime}-\beta^{2}} q\right), \\
l_{i}^{-} & =\partial_{-i} \\
\bar{l}_{i}^{0} & =q .
\end{aligned}
$$

where, $\lambda$ is a scale weight in the right sector of a primary field $\Phi$, and $q$ is its Lorentz weight. So far, we have described the "classical" symmetries of the model. Quantizing the model means calculating gravitational contribution to the "classical" action (2.2). This can be done in two ways: i) by direct Feynman graph calculation [18], which is interesting but tedious due to the

\footnotetext{
${ }^{5}$ Our notation can be compared to the one usually appearing in the literature [7] through the identification $J_{++} \rightarrow J^{(+)}, J_{+} \rightarrow J^{(0)}, J_{0} \rightarrow J^{(-)}$.
} 
non-local form of the action, or ii) requiring the persistence of the classical symmetries (3.2) at the quantum level [19]. This amounts to the requirement of the weakly vanishing of the appropriate generators $\widetilde{T}_{++}$and $T_{+-}$. We shall follow the second approach. The quantum analogue of the classical Sugawara form (3.15) is well known [20] and is given by

$$
\begin{aligned}
& T_{+-}=J_{0}\left(x^{+}\right) \\
& \widetilde{T}_{++}=\frac{1}{K+2} \eta^{a b}: J_{a} J_{b}:+\partial_{+} J_{+}+\frac{1}{K_{U(1)}}: \bar{J}_{+}^{2}:+\partial_{+} \bar{J}_{+} .
\end{aligned}
$$

where, " +2 " is the Casimir of the $S L(2, R)$ and " dots" mean appropriate normal ordering of the currents.

It is further possible to show that the quantum generators of the residual symmetry transformations satisfy the following OPE's

$$
\begin{aligned}
\widetilde{T}_{++}(x) \widetilde{T}_{++}(y)= & \frac{3 K /(K+2)-6 K+1-6 K_{U(1)}}{2\left(x^{+}-y^{+}\right)^{4}}+ \\
& \frac{2 \widetilde{T}_{++}(y)}{\left(x^{+}-y^{+}\right)^{2}}+\frac{\partial_{+} \widetilde{T}_{++}(y)}{x^{+}-y^{+}}+\text {reg. }, \\
\widetilde{T}_{++}(x) T_{+-}(y)= & \frac{2 T_{+-}(y)}{\left(x^{+}-y^{+}\right)^{2}}+\frac{\partial_{+} T_{+-}(y)}{x^{+}-y^{+}}+\text {reg. }, \\
T_{+-}(x) T_{+-}(y)= & \text { reg. . }
\end{aligned}
$$

It can be seen from (3.20) that the weakly vanishing of $\widetilde{T}_{++}$and $T_{+-}$is possible only if the total central charge, including matter and ghost contributions, is vanishing. This condition leads to the renormalized central charge through the equation

$$
\begin{aligned}
& n_{+}-28+c_{\text {grav. }}=0 \\
& c_{\text {grav. }}=\frac{3 K}{K+2}-6 K+1+24 Q_{\text {Lorent } z}^{2}
\end{aligned}
$$

where, $n_{+}$and -28 are respectively the chiral matter and the ghost contribution resulting from gauge fixing (3.1). Equations (3.21) give the renormalized 
Kac-Moody $S L(2, R)$ central charge

$$
K+2=\frac{\left(n_{+}-12+24 Q_{\text {Lorentz }}^{2}\right) \pm \sqrt{\left(n_{+}+24 Q_{\text {Lorentz }}^{2}\right)\left(n_{+}-24+24 Q_{\text {Lorentz }}^{2}\right)}}{12}
$$

From the equation (3.22) follows that the renormalized Kac-Moody $S L(2, R)$ central charge is dependent on a free parameter, through the $Q_{\text {Lorentz }}$, that can be adjusted at will to make a square root in (3.22) real for any value of $n_{+}$(see discussion in Section(5)). Therefore, contrary to the non-chiral gravity, in chiral models there are no forbidden regions corresponding to complex values of the central charge since it can always be made real. This is due to the presence of the Lorentz degree of freedom which acts as an additional " matter " contribution introducing, at the same time, a new dependence on a free parameter. Such a parameter has no fixed value in chiral models since there is no value that can remove the Lorentz anomaly.

\subsection{Generalization and extension to supersymmetric models}

In the previous subsection we have explained in detail the approach to the induced chiral gravity models. Prescriptions to obtain non-chiral results were given as well. Supersymmetric versions of the same models could be studied along the same lines but one has to have a detailed knowledge of the appropriate superspace structure model by model. Instead, we would like to present a simple, generalized, approach reproducing all the known results with little effort. It is based on the following observation. In order to maintain residual symmetries at the quantum level, in any model, one has to require the weakly vanishing of the residual symmetry generators leading to the vanishing of the total Virasoro central charge.

$$
c_{\text {matt. }}+c_{\text {grav. }}^{\mathrm{lc}}+c_{\text {ghosts }}^{\mathrm{lc}}=0,
$$

This is the formula which is subject to a simple generalization. $c_{\text {grav }}$. can be obtained from the equation (3.19) for an arbitrary group as

$$
c_{\text {grav. }}^{\mathrm{lc}}=\frac{K \operatorname{dim}(G)}{K+C_{v}}-6 K .
$$


where, $\operatorname{dim}(G)$ is the dimension of the appropriate $S L(2, R)$ group (or its supersymmetric version), $C_{v}$ is the corresponding Casimir, and $K$ is the renormalized Kac-Moody central charge of the same group. The generality of this expression stems from the fact that differences among various models are all contained within $c_{\text {matt. }}, c_{\text {ghosts, }}^{\text {lc }}$ while (3.24) takes into account the structure of the light-cone gauge group of residual symmetry. It works both for non chiral and chiral models since in the latter case the Lorentz degree of freedom acts as additional "matter" field, as far as $S L(2, R)$ group is concerned, and it will be contained in $c_{\text {matt. }}$. It will be explicitly shown later on.

Solving the algebraic quadratic equation (3.24) with respect to $K$ gives the gravitationally renormalized Kac-Moody central charge. If we denote the two roots $\kappa_{ \pm}=K_{ \pm}+C_{v}$ they will satisfy the following relation

$$
\begin{aligned}
\kappa_{+} \kappa_{-} & =a^{2} \\
\kappa_{+}+\kappa_{-} & =2 a-m Q_{l c}^{2} / 2
\end{aligned}
$$

We have introduced a parameter $m$ to explicitly stress the difference among various supersymmetric models: $m=1$ for $N=0,1$, and $m=2$ for $N=2$. From equations (3.23), (3.24) the parameters $a$ and $Q_{l c}$ are found to be

$$
\begin{aligned}
a^{2} & =C_{v} \operatorname{dim}(G) / 6 \\
Q_{l c}^{2} & =-\frac{1}{3 m}\left[c_{\text {matt. }}+c_{\text {ghosts }}^{\mathrm{lc}}+\left(\sqrt{6 C_{v}}-\sqrt{\operatorname{dim}(G)}\right)^{2}\right]
\end{aligned}
$$

In this way we get a simple evaluation of these parameters in terms of the characteristics of the residual symmetry group in the light-cone gauge. At this point it may be instructive to calculate these parameters for various models. First of all, let us calculate Casimir operators for various supersymmetric versions of the $S L(2, R)$ group. Its definition is

$$
C_{v} \delta_{a}^{b}=f_{a c d} f^{b c d}
$$

where, $f_{a b c}$ are anti-symmetric structure constants of the appropriate $S L(2, R)$ group. Using the values of these constants for $N=0,1,2$ one can find the general formula for the Casimir $C_{v}=2-N / 2$. For $\operatorname{dim}(G)=$ 
\#(bosonic generators) - \#(fermionic generators) we have values 3, 1, 0 respectively.

In order to calculate ghost contributions, let us look at the constraints of the residual symmetry generators. For $N=0$ they are $\widetilde{T}_{++}$and $T_{+-}$leading to the ghosts $\left(b_{++}, c_{-}\right)$and $\left(b, c_{+}\right)$, with contributions $-26,-2$ [ 8 ].

In the case $N=1$ the constraints are $\left(\widetilde{T}_{++}, G_{1 / 2+}\right),\left(T_{+-}, J_{1 / 2}\right)$. The appropriate ghosts are $\left(b_{++}, c_{-}\right),\left(b_{1 / 2+}, c_{1 / 2}\right),\left(b, c_{+}\right)$, and the single ghost $b_{1 / 2}$ with contributions $-26,11,-2,-1 / 2$ [9].

Subscript $1 / 2$ refers to the fermionic partner of the bosonic coordinate + . In the case $N=2$ the only difference with respect to the $N=1$ case is the doubling of the ghost and matter contribution due to additional supersymmetry [9]. Therefore, the counting of ghost contributions is $-26+2 \times 11-2 \times 2-2 \times(1 / 2)$.

\begin{tabular}{|c|c|c|c|}
\hline \hline & $N=0$ & $N=1$ & $N=2$ \\
\hline $\operatorname{dim}(G)$ & 3 & 1 & 0 \\
\hline$C_{v}$ & 2 & $3 / 2$ & 1 \\
\hline$a$ & 1 & $1 / 2$ & 0 \\
\hline$c_{\text {matt }}$ & $d$ & $3 d / 2$ & $3 d$ \\
\hline$c_{\text {ghost }}^{l c}$ & -28 & $-35 / 2$ & -9 \\
\hline$Q_{l c}^{2}$ & $(25-d) / 3$ & $(9-d) / 2$ & $(1-d) / 2$ \\
\hline \hline
\end{tabular}

Table 1: We display values of various parameters entering formulae (3.28) in the case of non-chiral models. Parameter $d$ refers to the number of scalar components, while left/right fermionic components are described by $n_{+/-}$. Supersymmetry imposes $n_{+}=d$ in the count of matter contribution, e.g. $d+n_{+} / 2=3 d / 2$. 


\begin{tabular}{|c|c|}
\hline \hline & $\kappa_{\mp}$ \\
\hline$N=0$ & $(d-13 \pm \sqrt{(d-1)(d-25)}) / 12$ \\
\hline$N=1$ & $(d-5 \pm \sqrt{(d-1)(d-9)}) / 8$ \\
\hline$N=2$ & {$[(d-1) \pm(1-d)] / 4$} \\
\hline \hline
\end{tabular}

Table 2: We display the values of the renormalized Kac-Moody central charge as defined by (3.26) with the help of the result in the previous Table. Notice that in the $N=2$ case the quadratic equation reduces to the linear one, and the absence of the square root means the absence of the complex values of the renormalized Kac-Moody central charge. This is due to the property $\operatorname{dim}(G)=0$ in this case.

The results for various models are presented in Table 1, while the values of the renormalized Kac-Moody central charge are given in Table 2

One of the nice features of the light-cone gauge is that one can also perform genuine perturbative calculations, due to the presence of a well defined regulator. This calculation serve as a check of the closed formulae displayed in the Table 2, as well as enable access to the "large $d$ " region, where stringy character of the induced gravity model is relevant. Perturbative calculations [18] are, however, complicated due to the non-local form of the effective action. Certain shortcuts can be exploited at the one-loop level, but the complication remains to higher level.

We would like to give a simple determination of the perturbative results (up to one-loop) based on our general equations (3.26). Classical Kac-Moody central charge $K_{\mathrm{cl}}$. is a coupling constant in the classical action (2.4) as can be seen from (3.17). From (3.26) one can see that only $\kappa_{+}$has a proper classical limit $c_{\text {matt }}>1$, which is $\kappa_{+} \equiv K_{c l}=c_{\text {matt }} / 6$, while $\kappa_{-} \sim O\left(1 / c_{\text {matt }}\right)$. One can perform perturbative calculations in $1 / K_{\mathrm{cl}}$, leading to the renormalized Kac-Moody central charge $K=Z_{K} K_{\text {cl. }}$. Equations (3.26), (3.28) allow a 
simple determination of the counterterm $Z_{K}$, which is found to be [18]

$$
Z_{K}=1+\frac{1}{c_{\text {matt. }}}\left(c_{\text {ghost }}^{\text {lc }}+\operatorname{dim}(G)\right)-\frac{6 \kappa_{-}}{c_{\text {matt. }}}
$$

From (3.30) one can see that all the higher loops contributions are hidden within $\kappa_{-}$.

\begin{tabular}{|c|c|}
\hline \hline & $Z_{K}$ \\
\hline$N=0$ & $1-(25 / d)$ \\
\hline$N=1$ & $1-(11 / d)$ \\
\hline$N=2$ & $1-(3 / d)$ \\
\hline \hline
\end{tabular}

Table 3: We display the one loop results for the Kac-Moody central charge counterterm following from (3.30). This results can be also obtained expanding results in Table 2 in powers of $1 / d$. Notice that $N=2$ case has only one-loop contributions. This is due to the fact that $\kappa_{-}=0$ in this case.

Another formula, that can be easily generalized and leads to a common treatment of various induced (super) gravity models, is the one following from the imposition of the weakly vanishing condition on the physical states within the BRST quantization procedure in the light-cone gauge. $\widetilde{T}_{++}$is the generator of the conformal transformations in the left moving sector, and is expressed through the Sugawara construction in terms of the $S L(2, R)$ currents (or their supersymmetrized version) as given by (3.15). One of these currents, i.e. $J^{0}$, describes, on the other hand, the scale transformation with weight $\lambda$ in the right moving sector as visible from (3.18). On general grounds [21], scale weight of the primary field, with respect to the scale transformations in the left sector, is $\Delta^{\text {left }}$, while with respect to the right sector it is $\Delta^{\text {right }}$. Therefore, in this particular case $\lambda \sim \Delta^{\text {right }}$. Applying 
the generator of the conformal transformations in the left moving sector on a primary field, and exploiting (3.15) one finds its conformal weight [19] to be

$$
\Delta^{\mathrm{left}}=\frac{\lambda(\lambda+1-N / 2)}{K+C_{v}}-\lambda,
$$

BRST analysis of the constraints of the residual symmetry has been performed in the light-cone gauge, and appropriate definitions of the physical states has been given in [22], [23]. Weakly vanishing of the generators of residual symmetry lead to the equation

$$
\bar{a}-\Delta_{0}=\frac{\lambda_{\beta}\left(\lambda_{\beta}+a\right)}{K+C_{v}}-\lambda_{\beta},
$$

where, $\bar{a}$ is the normal ordering ambiguity parameter of the BRST formalism and $\Delta_{0}$ is the scaling weight of the matter field. Equation (3.31) give the value of the parameter $a$ for various supersymmetric models as $a=1-N / 2$. Comparison to the Table 1 shows that the explicit values of $a$, as defined above, are equal to the ones defined by (3.27). Furthermore, we shall show later on that $a=\bar{a}$. Accordingly, from now on we shall be using only one symbol " $a$ ".

At this point we would like to mention that we are still working in full generality, in the sense that the results will apply both to chiral and non chiral (supersymmetric) models, since the presence of the (super)Lorentz field will be contained within $\Delta_{0}$ being just another "matter" field. An explicit example will be given later.

The subscript $\beta$ in $\lambda$ refers to the solution of the above equation in the presence of matter $\Delta_{0} \neq 0$. When $\Delta_{0}=0$ then $\beta \rightarrow \alpha$. The subscripts $\alpha, \beta$ have been introduced in order to make comparison to the conformal gauge results (which will follow) more transparent.

The roots of the algebraic quadratic equation (3.32) satisfy

$$
\begin{aligned}
& \lambda_{\beta_{+}} \lambda_{\beta_{-}}=\left(K+C_{v}\right)\left(\Delta_{0}-a\right) \\
& \lambda_{\beta_{+}}+\lambda_{\beta_{-}}=K+C_{v}-a
\end{aligned}
$$

The vacua will correspond to $\Delta_{0}=0$, or $\beta \rightarrow \alpha$, and are characterized by the scaling weights

$$
\begin{aligned}
& \lambda_{\alpha_{+}}=-a \\
& \lambda_{\alpha_{-}}=K+C_{v}
\end{aligned}
$$


Equations (3.34) show that there are always two distinct vacua described by the above $S L(2, R)$ weights, which are not, in general, projectively invariant. At this point we can establish relations between the renormalized Kac-Moody central charge and the above vacua as

$$
\kappa_{ \pm}=\frac{\lambda_{\alpha_{\mp}}^{2}}{\lambda_{\alpha_{-}}} .
$$

\section{Connection between the light-cone and conformal gauge}

In the conformal gauge the metric element takes on the form

$$
d s^{2}=e^{\varphi} d x^{+} d x^{-}
$$

Once this coordinate mesh is set over spacetime, the dynamics of the $\varphi$ is dictated by the Liouville action, and a residual invariance is present as a conformal symmetry in both left and right moving sectors. The gravitational interaction is represented by the Liouville potential with a coupling constant given by the non zero cosmological constant. Perturbative calculations are ambiguous in this gauge due to the lack of an appropriate regulator, as well as of a "small" parameter justifying a perturbative expansion [24]. As an alternative to perturbative calculations, the DDK approach [12], as well as the cocycle quantization [25], has been recently offered. The basic underlying idea in this approach is to replace the original, intractable, functional measure depending from the Weyl degree of freedom, with an invariant measure with respect to a background metric $\widehat{g}_{a b}$. The original metric is split as $g_{a b}=\widehat{g}_{a b} e^{\alpha \varphi}$. The Jacobian of the transformation is assumed to be local and of the same form as the original Liouville action, but with two unknown coefficients. One of them is the background charge $Q_{\text {conf }}$ and the other is the gravitational renormalization parameter $\alpha$ of the Liouville potential $e^{\alpha \varphi}$. The two free parameters are then fixed by the requirement of scale invariance with respect to the background metric $\widehat{g}_{a b}$. The above mentioned invariance leads to the conformal gauge equivalent of the light-cone equation (3.23), and is given by

$$
c_{\text {matt. }}+c_{\text {ghost }}^{\text {conf. }}+c_{\text {grav. }}^{\text {conf. }}=0
$$




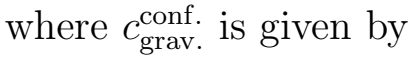

$$
c_{\text {grav. }}^{\text {conf. }}=m\left(1+3 Q_{\text {conf }}^{2}\right)+\frac{N}{2}
$$

Parameter $m$ enters the equation (4.3) due to the additive character of the central charge, and the fact that $N=2$ supersymmetry corresponds to doubling of the number of Weyl degrees of freedom. $N / 2$ is the contribution of the corresponding superpartners. The parameter $Q_{\text {conf }}$ obtained from the equation (4.2) is given by

$$
Q_{\text {conf }}^{2}=-\frac{1}{3 m}\left[c_{\text {matt }}+c_{\text {ghost }}^{\text {conf. }}+m+\frac{N}{2}\right]
$$

One could prove by explicit calculations that the $Q_{\text {conf }}$ given by (4.4) and $Q_{l c}$ given by (3.28) are identical. We shall give proof of this statement on general grounds later on.

The effect of Weyl dynamics in the conformal gauge is that the various classical operators will receive gravitational dressing described by the parameter $\beta$ in the Liouville potential $\Phi e^{\beta \varphi}$, where $\Phi$ represents conformal matter field. As we have mentioned earlier, the essence of the DDK approach consists of requiring the invariance under scale transformation with respect to the background metric $\hat{g}_{\mu \nu}$. Applying this condition to the Liouville potential in the presence of matter puts constraints on the total scale weights of such potential. These constraints can be determined in the general way by considering the scale weight of the super integration measure $d^{2} x d \theta^{+} d \theta^{-}$. Keeping in mind that the scale weight of the superspace parameters is $1 / 2$, one obtains the scale weight of the integration measure as $(-1+N / 2,-1+N / 2)$, which then gives the scale weight of the Liouville potential $(1-N / 2,1-N / 2) \equiv(\bar{a}, \bar{a})$. As stated earlier, this shows the equality $\bar{a}=a$, where $a$ is defined in (3.32). If we denote $\Delta_{0}$ the scale weight of the spinless matter field $\Phi$, then the scale weight of the Liouville potential is

$$
a-\Delta_{0}=-\frac{m}{2} \beta\left(\beta+Q_{\text {conf }}\right)
$$

Equivalent expression in the light-cone gauge is

$$
a-\Delta_{0}=\frac{\lambda_{\beta}\left(\lambda_{\beta}+a\right)}{K+C_{v}}-\lambda_{\beta}
$$


Confronting the above equation in case $\beta=\alpha$, e.g. $\Delta_{0}=0$, we obtain

$$
-\frac{m}{2} \alpha^{2}\left(1+\frac{Q_{\text {conf }}}{\alpha}\right)=\frac{\lambda_{\alpha}^{2}}{K+C_{v}}\left(1-\frac{K+C_{v}-a}{\lambda_{\alpha}}\right)
$$

from which we deduce the relation among various parameters in the two gauges

$$
-\frac{m}{2} \alpha^{2}=\frac{\lambda_{\alpha}^{2}}{K+C_{v}}
$$

and

$$
\frac{Q_{\text {conf }}}{\alpha}=-\frac{1}{\lambda_{\alpha}}\left(K+C_{v}-a\right)
$$

From equations (4.8), (4.9) one can prove the following relations

$$
Q_{\text {conf }}^{2}=-\frac{2}{m} \frac{\left(K+C_{v}-a\right)^{2}}{K+C_{v}}
$$

Further, exploiting relation (3.33) and the definitions of $\kappa_{ \pm}$one obtains the following relation

$$
\kappa_{+}+\kappa_{-}=2 a-m Q_{\text {conf }}^{2} / 2
$$

which proves the identity between $Q_{\text {conf }}$ and $Q_{l c}$ on general grounds. Comparing the explicit expression (4.9) and (3.28) we get a particularly simple relation between ghost contributions in the two gauges

$$
c_{\text {ghost }}^{\text {conf. }}=c_{\text {ghost }}^{\text {lc }}+\left(\sqrt{6 C_{v}}-\sqrt{\operatorname{dim}(G)}\right)^{2}-m-\frac{N}{2}
$$

Further identification of parameters in the two gauges can be obtained considering the equation (4.6) in the presence of matter $\left(\Delta_{0} \neq 0\right)$. In this case, one can rewrite equation (4.6), with the help of (4.8), (4.9), as

$$
-\frac{\beta}{\alpha} \lambda_{\alpha}+\frac{1}{K+C_{v}} \frac{\beta}{\alpha} \lambda_{\alpha}\left(a+\frac{\beta}{\alpha} \lambda_{\alpha}\right)=\frac{\lambda_{\beta}\left(\lambda_{\beta}+a\right)}{K+C_{v}}-\lambda_{\beta}
$$

which gives the following relations among various parameters

$$
\lambda_{\beta}=\frac{\beta}{\alpha} \lambda_{\alpha},
$$




$$
\begin{gathered}
\frac{Q}{\alpha_{ \pm}}=-1-\frac{\lambda_{\alpha_{\mp}}}{\lambda_{\alpha_{ \pm}}}, \\
\alpha_{ \pm}^{2}=-\frac{2 \lambda_{\alpha_{ \pm}}^{2}}{m \lambda_{\alpha_{-}}},
\end{gathered}
$$

Equations (4.15), (4.16) are the same as (4.8) and (4.9), which serves as a consistency check. We have dropped the subscripts ${ }_{\text {conf }}, l_{c}$, on the parameter $Q$ in view of the identity $Q_{\text {conf }} \equiv Q_{l c}$, as proven above.

\subsection{Particularity of the chiral models}

Chiral models are characterized by the presence of the dynamical Lorentz degree of freedom, which enters through the zweibein as the fundamental ingredient of the theory. The conformal gauge expression is

$$
e_{\mu}^{\hat{ \pm}}=\exp \left(\frac{\varphi \pm L}{2}\right) \delta_{\mu}^{\hat{x}}
$$

However, as far as DDK approach is concerned, the functional measure of the path integral for the dynamical fields is only dependent on the determinant of the zweibein and, therefore, independent of the Lorentz degree of freedom. So, prescription of defining a modified (proper) measure, will go unchanged in the chiral case due to the fact that the line element is Lorentz independent [26]. In this respect, Lorentz field appears to be just additional matter field in the theory. Its scale weight can be obtained considering its OPE with the generator of the conformal transformation as given by the component $T_{ \pm \pm}$ of the energy momentum tensor following from (2.6), which are

$$
\begin{aligned}
& T_{++}=\left(\partial_{+} \varphi\right)^{2}-2 Q \partial_{+}^{2} \varphi+\left(\partial_{+} \chi\right)^{2}-2 Q_{\mathrm{Lorentz}}^{\text {left }} \partial_{+}^{2} \chi \\
& T_{--}=\left(\partial_{-} \varphi\right)^{2}-2 Q \partial_{-}^{2} \varphi+\left(\partial_{-} \chi\right)^{2}-2 Q_{\mathrm{Lorentz}}^{\text {right }} \partial_{-}^{2} \chi
\end{aligned}
$$

where, we have used a redefinition of the Lorentz field as $\chi=L+\alpha \beta \varphi /(b-$ $\left.\beta^{2}\right)$. The presence of $Q_{\mathrm{Lorentz}}^{\text {left } / \text { ight }}$ indicates that there is a background Lorentz charge in the theory, and it is different in the left/right sector as can be seen 
from the expressions

$$
\begin{aligned}
Q_{\text {Lorentz }}^{\text {left }} & \equiv\left(b-\beta^{2}\right)^{1 / 2}\left(\frac{\alpha \beta}{b-\beta^{2}}+1\right) \\
Q_{\text {Lorentz }}^{\text {right }} & \equiv\left(b-\beta^{2}\right)^{1 / 2}\left(\frac{\alpha \beta}{b-\beta^{2}}-1\right)
\end{aligned}
$$

Lorentz field will enter the Liouville potential in the exponential form as $e^{\kappa \chi}, e^{\tilde{\kappa} \chi}$ where $\kappa, \tilde{\kappa}$ are holomorphic (left), and anti-holomorphic (right) momenta of the Lorentz field. They are introduced due to the fact the Lorentz field carries spin, i.e. couples to the spin connection and therefore one has to distinguish between left and right components. Its scale dimension, in the left sector, is given by

$$
\Delta_{\text {Lorentz }}^{\text {left }}=-\frac{m}{2} \kappa\left(\kappa+Q_{\text {Lorentz }}^{\text {left }}\right)
$$

and an analogous equation is valid in the right sector substituting $\kappa \rightarrow \tilde{\kappa}$ and $Q_{\text {Lorentz }}^{\text {left }} \rightarrow Q_{\text {Lorentz }}^{\text {right }}$. The presence of spin is also visible through the fact $Q_{\text {Lorentz }}^{\text {left }} \neq Q_{\text {Lorentz }}^{\text {right }}$. In fact, from the definition of spin $s=\Delta^{\text {right }}-\Delta^{\text {left }}$, and from the above equations one finds $s=(\kappa m / 2)\left(Q_{\text {Lorentz }}^{\text {left }}-Q_{\text {Lorentz }}^{\text {right }}\right) \neq 0$ We have assumed simplified case of $\tilde{\kappa}=\kappa$ which gives a simple looking formula. It can be shown, in general, that the dressing operators with spin are necessary in chiral theories in order to satisfy momentum conservation rules in the presence of the background charges and obtain non-trivial correlation functions. Such operators are discussed in [26] and we refer reader for details to it. We repeat just notions necessary for our purpose. Given a matter operator of conformal weight $\left(\Delta^{\text {left }}, \Delta^{\text {right }}\right)$, it will acquire gravitational dressing of the form $e^{\alpha \varphi} e^{\kappa \chi}$ in the left sector (analogous situation in the right sector). We shall assume that the Weyl field $\varphi$ carries no spin as in the non chiral case in order to be able to reproduce a local operator analog of the cosmological constant. In order to make a dressed operator of $(a, a)$ scale weight, it has to satisfy the analog of the equation (4.5) in the chiral case

$$
a-\Delta_{0}^{\text {left }}-\Delta_{\text {Lorentz }}^{\text {left }}=-\frac{m}{2} \beta\left(\beta+Q_{\text {conf }}\right)
$$

Analogous extension of the light-cone equation (4.6) is

$$
a-\Delta_{0}=\frac{\lambda_{\beta}\left(\lambda_{\beta}+a\right)}{K+C_{v}}-\lambda_{\beta}+\frac{q_{\beta}^{2}}{K_{U(1)}}-q_{\beta}
$$


Comparing these two equations in the same way followed in the non-chiral case we obtain the relations (4.14), (4.15), (4.16), and, in addition, relations between the appropriate parameters characterizing the Lorentz field in the two gauges

$$
\begin{gathered}
\frac{Q_{\text {Lorentz }}}{k_{\alpha}}=-\frac{K_{U(1)}}{q_{\alpha}}, \\
q_{\beta}=\frac{k_{\beta}}{k_{\alpha}} q_{\alpha} \\
k_{\beta}^{2}=-\frac{2 q_{\beta}^{2}}{m K_{U(1)}}
\end{gathered}
$$

where, $k_{\beta}$ is the gravitational dressing parameter of the Lorentz field in presence of matter. In principle, one can consider quantum operators with scale weight $\left(a^{\text {left }}, a^{\text {right }}\right)$ with $a^{\text {left }} \neq a^{\text {right }}$ which are quantum analogs of classical operators involving zweibein and its gravitational covariant derivatives. Therefore, in chiral theories the choice of dressing operators is richer than in non-chiral case. However, these are necessarily non-local operators. In order to reproduce a local operator (cosmological constant) as in non-chiral case absence of matter requires $\Delta_{0}=0$ and $\Delta_{\text {Lorentz }}=0$. This gives values $\kappa_{\alpha}=0$, and $\kappa_{\alpha}=-Q_{\text {Lorentz }}$ in the conformal gauge, or $q_{\alpha}=0$ and $q_{\alpha}=K_{U(1)}$. Therefore, we see that in the chiral gravity vacuum states are both scale and Lorentz non-invariant.

\subsection{Derivation of KPZ equation}

The main effect of gravitational dynamics is to renormalize flat space quantities. Among them a particular interest is in calculating gravitational effects on the naive flat space scaling dimensions of various fields in the theory [19]. One approach is to notice that the gravitational effects will violate conformal block decomposition of the multipoint functions. Exploiting differential equations that these functions satisfy in conformal field theory, one can obtain the above mentioned renormalized values of the scaling dimensions. This works fine as long as one works with projectively invariant vacua. Unfortunately, models described in this paper have non-invariant vacua and only hand-waving arguments [22] lead to the determination of the scaling dimensions. On the other hand, in the DDK approach a variation of the area, i.e. 
cosmological constant, with respect to the background scale transformations enable one to determine a renormalized scaling dimension in terms of parameters $\alpha, \beta$ [27]. At this point we would like to mention that the DDK derivation of the renormalized scaling dimension would go unchanged in the presence of the Lorentz field. This is due to the fact that the background scale transformation affects only Weyl degree of freedom, and leaves Lorentz field unchanged. That is way it is considered just another "matter" field.

Therefore, starting from the definition of the gravitational scaling weight, as obtained in the conformal gauge,

$$
\Delta=a\left(1-\frac{\beta}{\alpha}\right)
$$

and exploiting relation (4.14) one can write the above weight in the light-cone gauge as

$$
\Delta=a\left(1-\frac{\lambda_{\beta}}{\lambda_{\alpha}}\right)
$$

Equation (4.29) indicate that one is actually referring to the renormalized scaling weight in the right sector $\Delta=\Delta^{\text {right }}$ since this one is related to the scale weight $\lambda$. Such a specification was not important in the non-chiral case since matter fields are spinless. As we have shown in the chiral case, we have to require $\Delta^{\text {left }} \neq \Delta^{\text {right }}$. Inserting (4.29) into (3.32) leads to the KPZ equation for the non-chiral models

$$
\Delta-\Delta_{0}=\kappa \frac{\Delta}{a}\left(\frac{\Delta}{a}-1\right)
$$

In the case of chiral models, the above equation becomes

$$
\Delta^{\text {right }}-\Delta_{0}^{\text {left }}=\kappa \frac{\Delta^{\text {right }}}{a}\left(\frac{\Delta^{\text {right }}}{a}-1\right)+\frac{q_{\beta}^{2}}{K_{U(1)}}-q_{\beta}
$$

Similar equation has been found in the reference [28], but with an error in identifying the Lorentz scale dimension.

With the identifications (4.27) one sees that the KPZ equations in the chiral and non-chiral case are essentially the same with the Lorentz field being formally absorbed in the substitution $\Delta_{0}^{\text {left }} \rightarrow \Delta_{0}^{\text {left }}+\Delta_{\text {Lorentz. }}^{\text {left }}$ With this in 
mind, we can continue the general discussion of the renormalized gravitational scaling dimensions.

We have at disposal four states labelled by $\lambda_{\alpha_{ \pm}}$and $\lambda_{\beta_{ \pm}}$. Therefore, we can define four different renormalized scaling dimensions satisfying the above KPZ equation:

$$
\begin{aligned}
& \widehat{\Delta}_{ \pm}=a\left(1-\frac{\lambda_{\beta_{ \pm}}}{\lambda_{\alpha_{ \pm}}}\right) \\
& \Delta_{ \pm}=a\left(1-\frac{\lambda_{\beta_{\mp}}}{\lambda_{\alpha_{ \pm}}}\right)
\end{aligned}
$$

Equation (4.33) has a non-trivial limit $\Delta_{0} \rightarrow 0$ (i.e. $\lambda_{\beta_{ \pm}} \rightarrow \lambda_{\alpha_{ \pm}}$) which allows the definition of a non-trivial string susceptibility coefficient as the renormalized scaling dimensions of the vacuum $\left.\Delta_{ \pm}\right|_{\Delta_{0}=0}=\Gamma_{ \pm}$. Accordingly, we find

$$
\Gamma_{+}=\lambda_{\alpha_{-}}-\lambda_{\alpha_{+}}
$$

and

$$
\Gamma_{-}=a \frac{\Gamma_{+}}{\Gamma_{+}-a}
$$

\begin{tabular}{|c|c|c|}
\hline \hline & $\Gamma_{+}$ & $\Gamma_{-}$ \\
\hline$N=0$ & $K+3$ & $(K+3) /(K+2)$ \\
\hline$N=1$ & $K+2$ & $(K+2) /(2 K+3)$ \\
\hline$N=2$ & $K+1$ & 0 \\
\hline \hline
\end{tabular}

Table 4: We display the values of the string susceptibilities coefficients as described by (4.34), (4.35).

In order to connect our result with the spectrum of physical states, we assume that any physical state $|\lambda\rangle$, characterized by the appropriate $S L(2, R)$ 
scaling dimension $\lambda$, is created out of a vacuum $\left|\lambda_{\alpha}\right\rangle$ by the action of some operator $O$. The scaling dimension of this operator $O$ is given by $\delta=\lambda-\lambda_{\alpha}$. Therefore, we can define four possible scaling operators $\widehat{O}_{ \pm}, O_{ \pm}$with appropriate dimensions

$$
\begin{aligned}
& \widehat{\delta}_{ \pm}=\lambda_{\beta_{ \pm}}-\lambda_{\alpha_{ \pm}}, \\
& \delta_{ \pm}=\lambda_{\beta_{ \pm}}-\lambda_{\alpha_{\mp}}
\end{aligned}
$$

From the general relations (3.33) one can further prove

$$
\begin{aligned}
& \delta_{+}=-\delta_{-} \\
& \widehat{\delta}_{+}=-\widehat{\delta}_{-},
\end{aligned}
$$

then, the following relations are established

$$
\begin{aligned}
& \Delta_{ \pm}=-\frac{\lambda_{\alpha_{+}}}{\lambda_{\alpha_{ \pm}}} \delta_{ \pm} \\
& \widehat{\Delta}_{ \pm}=\frac{\lambda_{\alpha_{+}} \widehat{\delta}_{ \pm}}{\lambda_{\alpha_{ \pm}}} .
\end{aligned}
$$

The string susceptibility results to be

$$
\Gamma_{ \pm}=\frac{\lambda_{\alpha_{+}}}{\lambda_{\alpha_{ \pm}}}\left(\widehat{\delta}_{ \pm}-\delta_{ \pm}\right)
$$

In order to display the action of various operators, whose scaling dimensions are given by the above expressions, let us introduce the following diagram:

$$
\begin{array}{llll}
\left|\lambda_{\beta_{+}}\right\rangle & \stackrel{O_{+} \widehat{O}_{+}}{ } & \left|\lambda_{\beta_{-}}\right\rangle \\
\widehat{O}_{+}=\widehat{O}_{\Delta_{+}} & \uparrow & \uparrow \widehat{O}_{-}=\widehat{O}_{+}^{-1} \\
& \stackrel{O_{\Gamma_{+}}=\widehat{O}_{+} O_{+}^{-1}}{\longrightarrow} & \left|\lambda_{\alpha_{-}}\right\rangle
\end{array}
$$

Now, it is possible to interpret the plethora of definitions given by the earlier formulae. First of all, the existence of two different $S L(2, R)$ vacua 
divides physical states into a "+" and a "-" sectors. The scale operators that induce transition in the each sector separately are the hatted ones . The transitions between different sectors is achieved by the un-hatted operators along the diagonals of the diagram, or by a combination of hatted and un-hatted operators along the sides. In particular the string susceptibility $\Gamma_{+}$is represented in the above scheme as the scale dimension of an operator that connects the different vacua. In other words, the existence of non-degenerate $S L(2, R)$ vacua implies non-trivial string susceptibility and, therefore, phase transitions in the appropriate theory of gravity. On the contrary, the corresponding hatted quantity is trivially zero since acting in the same sector. In view of the additive character of the scaling dimension, they are truly given by the definitions (4.36), (4.37). The "scaling" dimensions $\Delta$ 's, satisfying KPZ equation, are equal to true scaling dimensions $\delta$ 's only for "+" quantities (hatted and un-hatted) as can be seen in (4.42), (4.42). On the other hand, $\Delta_{-}, \widehat{\Delta}_{-}$and, as a consequence, $\Gamma_{-}$, cannot be interpreted in terms of the simple scheme given above because, as can be seen from (4.42), (4.42), (4.43), they are multiplicatively related to $\delta$ 's and $\widehat{\delta}$ 's, while the property of genuine scale dimensions is their additivity. It may be interesting to compare our approach with the recently proposed modified matrix models [29, [30]. From our vantage point, the effect of the fine tuning of the interaction in the matrix models can be interpreted as a switching from the transition connecting the vacuum, say $\left|\lambda_{\alpha_{+}}\right\rangle$, to the state $\left|\lambda_{\beta_{+}}\right\rangle$, to the transition from the same vacuum to another state $\left|\lambda_{\beta_{-}}\right\rangle$. In other words, the effect of additional interaction in modified matrix models amounts to the exchange $\left|\lambda_{\beta_{+}}\right\rangle \longleftrightarrow\left|\lambda_{\beta_{-}}\right\rangle$.

Although we can also reproduce the modified string susceptibility coefficient given by (4.35), we are not able to give it an interpretation within our scheme because of the difficulty mentioned above.

\section{Restrictions on various parameters}

\subsection{Non-Chiral models}

It is known that in the conformal gauge the parameters of the model should satisfy certain conditions. One of them is the requirement of reality of the parameter $\alpha$ in order to correctly define the scale transformation with respect 
to the background metric. The expression for $\alpha$ is obtained from (4.5) and is given by

$$
\alpha_{ \pm}=\frac{-\sqrt{m Q^{2}} \pm \sqrt{m Q^{2}-8 a}}{2 \sqrt{m}}
$$

Requirement of reality of $\alpha$ reduces to the condition $m Q^{2}-8 a \geq 0$. By using the explicit form of $Q_{\text {conf }}$ one finds the condition

$$
0 \geq c_{\text {matt. }}+c_{\text {ghost }}^{\text {conf. }}+24 a+m+\frac{N}{2}
$$

\begin{tabular}{|c|c|}
\hline \hline & $d$ \\
\hline$N=0$ & $\leq 1$ \\
\hline$N=1$ & $\leq 1$ \\
\hline$N=2$ & $\leq 1$ \\
\hline \hline
\end{tabular}

Table 5: We display the restriction on the number of scalar fields $d$ (" dimension " of the appropriate model). It shows the well known $d \leq 1$ barrier, characteristic of the DDK approach. Warning: $d$ is not always identical to $c_{\text {matt }}$, as explained earlier.

For transparency we give the restrictions on the number of matter fields, in various non-chiral supersymmetric models, which follow from the above condition in Table 5. Secondly, one can show, by an appropriate rescaling, that the kinetic term of the Liouville field is multiplied by $Q^{2}$, so that it results to be physical only if $Q^{2}$ is positive [3]. This condition translates into the following equation

$$
0 \geq c_{\text {matt. }}+c_{\text {ghost }}^{\text {conf. }}+m+\frac{N}{2}
$$




\begin{tabular}{|c|c|}
\hline \hline & $d$ \\
\hline$N=0$ & $\leq 25$ \\
\hline$N=1$ & $\leq 9$ \\
\hline$N=2$ & $\leq 1$ \\
\hline \hline
\end{tabular}

Table 6: We display the restrictions on the parameter $d$ which follows from (5.3). It is clear that the condition displayed in Table 5 are more stringent and represent a common solution for (5.2), (5.3).

Explicit results satisfying the above condition are given in Table 6.

The condition (5.2) is more stringent and gives the common solution for both (5.2), (5.3). It is known as " $d=1$ barrier " which restricts the application of the DDK approach to minimal models only.

On the other hand, in the light cone gauge the relevant parameter is the renormalized Kac-Moody central charge which must be real. Equations (4.16) and (3.35) relate this central charge to $\alpha^{2}$. However, real $\alpha^{2}$ can be obtained by two possible choices for $\alpha$ :

i) $\alpha$ real. This condition has been analyzed above, and the corresponding induced gravity models can be described in terms of minimal models;

ii) $\alpha$ imaginary. Then, the Liouville field becomes ghostlike in the conformal gauge [12], and its physical meaning turns out to be unclear. On the contrary, this condition is still allowed in the light-cone gauge, as discussed above. In this case $Q^{2}<0$, which leads to the condition

$$
0 \leq c_{\text {matt. }}+c_{\text {ghost }}^{\text {conf. }}+m+\frac{N}{2}
$$

As before, the range of explicit values is given in Table 7 . Equation (5.4) enables one to access the region of large $c_{\text {matt. }}$, where the stringy character of induced gravity is relevant and the inverse Kac-Moody central charge 


\begin{tabular}{|c|c|}
\hline \hline & $d$ \\
\hline$N=0$ & $\geq 25$ \\
\hline$N=1$ & $\geq 9$ \\
\hline$N=2$ & $\geq 1$ \\
\hline \hline
\end{tabular}

Table 7: We display the restrictions on the parameter $d$ which follows from (5.4). We can see that in $N=2$ case there is no intermediate region, showing the absence of complex values of the Kac-Moody central charge, as argued earlier.

is small enough to allow reliable perturbative calculations in the light-cone gauge.

\subsection{Specific features of the chiral models}

At this point we would like to apply the above general formulae to chiral (super) gravity models. The underlying motivation is the presence of the Lorentz degree as an additional, dynamical, component of the gravitational field arising from the breaking of the $U(1)$ Lorentz symmetry, and as such these models are different from previously analyzed non chiral models. In particular, there is a free parameter in this theory as a result of the impossibility to remove the Lorentz anomaly. It is hidden within $Q_{\text {Lorentz }}$ as defined by (3.12). This formula can be rewritten in terms of the parameters of the asymmetric action (2.4), with the help of relations (2.5), as

$$
3 m Q_{\text {Lorentz }}^{2}=\frac{\hat{b}}{4}+\frac{\Delta \mathcal{N}}{2}+\frac{\Delta \mathcal{N}^{2}}{4 \hat{b}} .
$$

\footnotetext{
${ }^{6}$ For consistency, we mention the relation between $Q_{\text {Lorentz }}$ as defined in (5.5) and in (3.12) $3 m Q_{\text {Lorentz }}^{2} \rightarrow 48 \pi Q_{\text {Lorent } z}^{2}$. With this rescaling one gets rid of factors $\pi$ following from (2.5).
} 
As we have already clarified in the previous discussion, the contribution of the Lorentz field will be contained within $c_{\text {matt. }}$ which is now given by

$$
c_{\text {matt. }}^{\text {chir. }}=\frac{\mathcal{N}-\Delta \mathcal{N}}{2}+m\left(1+3 Q_{\text {Lorentz }}^{2}\right)+\frac{N}{2}
$$

$\mathcal{N}$ and $\Delta \mathcal{N}$ will contain the contributions of the (super) matter fields, and $N / 2$ is the contribution of the super-partners of the Lorentz degree of freedom.

With the above definitions, (5.2) reduces to a quadratic equation in the parameter $\hat{b}$. The roots of that equation are given by

$$
\begin{aligned}
\hat{b}_{ \pm}=- & {\left[\left(\frac{\mathcal{N}+\Delta \mathcal{N}}{2}+c_{\text {ghost }}^{\text {conf }}+24 a+N+2 m\right)^{1 / 2} \pm\right.} \\
& \left.\left(\frac{\mathcal{N}-\Delta \mathcal{N}}{2}+c_{\text {ghost }}^{\text {conf }}+24 a+N+2 m\right)^{1 / 2}\right]^{2}
\end{aligned}
$$

In the same way (5.3) has roots

$$
\begin{aligned}
& \hat{b}_{ \pm}=- {\left[\left(\frac{\mathcal{N}+\Delta \mathcal{N}}{2}+c_{\text {ghost }}^{\text {conf }}+N+2 m\right)^{1 / 2} \pm\right.} \\
&\left. \pm\left(\frac{\mathcal{N}-\Delta \mathcal{N}}{2}+c_{\text {ghost }}^{\text {conf }}+N+2 m\right)^{1 / 2}\right]^{2}
\end{aligned}
$$

One can see that the equations (5.4), (5.2), (5.3), can be always satisfied for any $\mathcal{N}, \Delta \mathcal{N}$, due to the arbitrariness of $\hat{b}$. For the case $N=0, \mathcal{N}$ and $\Delta \mathcal{N}$ are given by (2.5). It can be easily generalized to contain also supersymmetric matter contributions in the following way

$$
\mathcal{N}=m d+\frac{m}{2}\left(n_{+}+n_{-}\right)
$$

and

$$
\Delta \mathcal{N}=\frac{m}{2}\left(n_{-}-n_{+}\right)
$$

where, $d$ represents the contribution of the scalar fields, $n_{+}$is the contribution of the left chirality fermions, and $n_{-}$is the contribution of the right chirality fermions. Supersymmetry forces $n_{+}=d$.In order to have genuine chiral models, we assume that the number of heterotic (right chirality) fermions is 
different from the one given in the heterotic string models. This is necessary in order to have a Lorentz anomaly. So to speak, we are considering offcritical dimensions heterotic string models described as chiral gravity models. In order to be coherent with the notation in the non-supersymmetric case (2.5) we assumed that, in that case, fermions are Majorana fermions, while superfield contains Weyl-Majorana fermions. This is why we have $n_{ \pm} \rightarrow 2 n_{ \pm}$ in the formula (2.5)

Explicit calculations show that the equation (5.7) depends only on the matter contribution. This leads to a very simple expression for the conformal gauge ghost contribution

$$
c_{\text {ghost }}^{\text {conf }}=-24 a-2 m-N
$$

With the help of the equation (4.12), one can find an equally simple expression for the ghost contribution in the light-cone gauge

$$
c_{\text {ghost }}^{\text {lc }}=-\left(\sqrt{6 C_{v}}+\sqrt{\operatorname{dim}(G)}\right)^{2}-m-\frac{N}{2}
$$

These equations give an amazingly simple way to calculate ghost contribution with no need to study ghost superfield structure model by model. All one needs to know is the structure of the residual (super) $S L(2, R)$ symmetry of the light-cone gauge. With the help of (5.11) equations (5.8), (5.7) are written as

$$
\begin{aligned}
& \hat{b}_{ \pm}=-\left[\left(\frac{\mathcal{N}+\Delta \mathcal{N}}{2}\right)^{1 / 2} \pm\left(\frac{\mathcal{N}-\Delta \mathcal{N}}{2}\right)^{1 / 2}\right]^{2} \\
& \hat{b}_{ \pm}=-\left[\left(\frac{\mathcal{N}+\Delta \mathcal{N}}{2}-24 a\right)^{1 / 2} \pm\left(\frac{\mathcal{N}-\Delta \mathcal{N}}{2}-24 a\right)^{1 / 2}\right]^{2}
\end{aligned}
$$

The equation (5.13) corresponds to zeroes of the quadratic equation in $\hat{b}$ that should satisfy the requirement of real $\alpha$, as in (5.2), for chiral models. First, we realize that there are real zeros for any choice of matter fields and the inequality (5.2) can be fulfilled. But, $\hat{b}$ is always negative. For the equation (5.7) the previous analysis goes through unchanged and the conclusion is that the inequalities (5.3) (5.4) can be satisfied as well. However, in this case $\hat{b}$ can be either positive or negative depending on the choice of matter fields. Therefore, there is a common solution for the inequalities (5.3), 
(5.2) with $\hat{b}<0$, while inequality (5.4) can be satisfied with both $\hat{b}>0$, $\hat{b}<0$. The meaning of these choices will be discussed in the conclusions. Nevertheless, renormalized Kac-Moody central charge is always real in chiral models contrary to the non-chiral situation.

\section{Discussion of the results}

In this paper we have concentrated on the description of the parameters characterizing the two-dimensional light-cone structure of induced gravity, in order to take advantage of the underlying $S L(2, R)$ residual symmetry. We have given a general description of the equations containing all the important information about the theory. In this way, we were able to summarize all the chiral and non chiral models, as well as their supersymmetric versions, within two basic formulae written in terms of two fundamental parameters expressible through the characteristic quantities of the residual symmetry group. The motivation for such a general approach is to have a compact and simple expression containing all the relevant information about induced gravity models. At every step we have however stressed and discussed the basic difference among chiral and non-chiral versions of the model. The motivation was to investigate alternative ways of avoiding the region of complex values of the renormalized Kac-Moody central charge.

We have found that the chiral models, due to the presence of the Lorentz anomaly, offer a new possibility with respect to the non chiral models. This is due to the presence of an arbitrary parameter, which stems from a chiral theory because no regularization, i.e. fixing of the parameter, can restore the classical symmetry 14. So far, the treatment of induced gravity models was mainly restricted to a particular gauge choice. Comparison between the two gauges has been given in more detail in [3], as far as we know. Therefore, we considered it useful to establish detailed connection among the parameters characterizing the two gauges. This could be useful when considering the strong gravity region in the conformal gauge. Once we have established connection between the parameters of the light-cone gauge and the corresponding parameters in the conformal gauge, we see that the reality of the string susceptibility in conformal gauge is equivalent to the reality of the renormalized Kac-Moody central charge in light-cone gauge. The presence of an arbitrary parameter in chiral models enables us to get rid of complex 
values of Kac-Moody central charge for any choice of matter fields. On the contrary, in non-chiral models only $N=2$ supergravity model offers such a possibility due to the particular structure of the residual $S L(2, R)$ symmetry, i.e. $\operatorname{dim}(G)=0$. Furthermore, we have analyzed restrictions on various parameters in both gauges, and found that the condition imposed in the conformal gauge can be satisfied with the choice of parameter $\hat{b}<0$ only. This would be the chiral analog of the $d=1$ barrier for the non-chiral models. We have also found that the stringy region (large $d$ ) of the chiral induced gravity can be reached by the choice of the parameter $\hat{b}>0$. This would be the analog of the situation in the non-chiral models, where DDK conformal gauge approach is limited to the minimal models only, while the light-cone approach enables to investigate the large $d$ region as well, since the Kac-Moody central charge is real there too. This would be the end of the parallel between chiral and non-chiral models if there were no further, independent, restrictions on the parameter $\widehat{b}$. However, if one looks at the structure of the Lagrangian (2.2) written in terms of the dynamical components in the light-cone gauge, one can see that the parameter $\widehat{b}$ figures as a coupling constant of the kinetic term of the Lorentz field. Therefore, apart from the requirement of the reality of various physical parameters, one has additionally to require the correct sign of the kinetic term of the Lorentz field to make it physical. Such a requirement puts an additional constraint on the free parameter $\hat{b}$. In fact, in order for the Lorentz field to be physical one has to require $\hat{b}>0$. Comparing this constraint with the independent one following from equation (5.13), which guarantees the reality of Kac-Moody central charge, we see that a consistent chiral gravity model, can only be achieved for the particular chiralities assignment

$$
\frac{\mathcal{N} \pm \Delta \mathcal{N}}{2} \leq 24 a
$$

For sake of transparency, we present the following table of explicit results for various chiral models. The meaning of this result is the following: although

it is possible to get rid of complex values of the renormalized Kac-Moody central charge without restrictions on the number of chiralities, further requirement of positive kinetic energy for the physical degrees of freedom, nevertheless restricts the number of chiralities, as it is described above. In this way, it is possible to have sensible chiral gravity models in spite of their 


\begin{tabular}{|c|c|c|}
\hline \hline & $n_{+}=d$ & $n_{-}$ \\
\hline$N=0$ & $\leq 24$ & $\leq 24-n_{+}$ \\
\hline$N=1$ & $\leq 12$ & $\leq 12-n_{+}$ \\
\hline$N=2$ & $\leq 0$ & $\leq-n_{+}$ \\
\hline
\end{tabular}

Table 8: We list regions of allowed values of chiralities leading to the consistent (with physical Lorentz degree of freedom) chiral models except in the case $N=2$.

anomalous character and, at the same time, to avoid the unpleasant region of complex values of renormalized Kac-Moody central charge. With respect to the previous discussion, regarding parameter $\hat{b}$, we can now say the following: additional requirement of a physical character of the Lorentz field points to the applicability of the chiral models to the region of large $d$ where its stringy character is relevant, and where reasonable perturbative calculations can be performed. $N=2$ case has a particular property: the appropriate non-chiral model gives real Kac-Moody central charge for any value of $d$, while the chiral version is not consistent, i.e. in this case Lorentz field is ghostlike.

We have also found a useful generalized way of extracting essential information about various aspects of induced gravity models. Previously, one had to control the details of each model in terms of its (super)field structure, (super)current, (super)ghosts, etc. We have been able to give simple formulae, not previously present in the literature, where the above mentioned work is reduced to a minimum, and all one needs to know is the structure of the group of residual symmetry in the light-cone gauge in order to reproduce all known results. Furthermore, we have established relations among various parameters in the light-cone and conformal gauges, which enable us to reproduce also the known conformal gauge results. As a byproduct of the above results, we have given a simple derivation of the KPZ equation in both chiral 
and non-chiral situations, which led to the expression of the renormalized gravitational scaling dimension, and therefore of the critical exponents, in terms of the $S L(2, R)$ scale weights of the vacuum state. In this picture the string susceptibility is described, at least in the light-cone gauge language, as the scale weight of an operator interpolating between the $S L(2, R)$ vacua states, whose multiplicity gives origin to the phase transitions in the induced gravity models. In this framework we have reproduced recent results of the modified matrix models.

\section{References}

[1] A.M.Polyakov Phys. Lett. 103B, (1981), 207

[2] J-L.Gervais, A.Neveu Nucl. Phys. bf B238, (1984), 396

[3] A.A.Tseytlin Int. J. Mod. Phys. Lett. A5, (1990), 1833

[4] T.Curtright, C.Thorne Phys. Rev. Lett. 48, (1982), 309;

J.Gervais, A.Neveu Nucl. Phys. bf B199, (1982), 59

[5] J-L.Gervais, J.Schnittger Phys. Lett. 315B, (1993), 258

J-L.Gervais, J-F.Roussel Nucl. Phys. bf B426, (1994), 140

[6] H.Dorn, H-J.Otto in Proceed. XXVIII In. Symp. on the Theory of Elementary Particles Wendisch-Rietz, August 1994

[7] A.M.Polyakov Mod. Phys. Lett. A2, (1987), 893

[8] V.G.Knizhnik, A.M.Polyakov, A.B.Zamolodchikov Mod. Phys. Lett. A3, (1988), 819

[9] A.M.Polyakov, A.B.Zamolodchikov Mod. Phys. Lett. A3, (1988), 1213

Z.Yang Phys. Lett. B245, (1990), 92

Riu-Ming Xu Phys. Lett. B247, (1990), 295

[10] R.Jackiw, R.Rajaraman Phys. Rev. Lett. 54, (1985), 1219

[11] A.Smailagic Phys. Lett. B195, (1987), 213 
[12] J.Distler, H.Kawai Nucl. Phys. B321, (1989), 509;

F.David Mod. Phys. Lett. A3, (1988), 1651

[13] S.J.Gates jr., M.T.Grisaru, L.Mezincescu, P.K.Townsend Nucl. Phys. B286, (1986), 1;

A.Smailagic, E.Spallucci Class. and Quant. Grav. 10, (1993), 451;

[14] A.Smailagic, E.Spallucci Phys. Lett. B284, (1992), 17

[15] H.Leutwyler Phys. Lett. B153, (1985), 65

[16] A.H. Chamseddine, M. Reuter Nucl. Phys. B317, (1989), 757

[17] A.Smailagic, E.Spallucci Phys. Lett. B317, (1993), 526

[18] K.A.Meissner, J.J.Pawelczyk Mod. Phys. Lett. A5, (1990), 763

A.Smailagic, Mod. Phys. Lett. A5, (1990), 125

G.W.Delius, M.T.Grisaru, P.van Nieuwenhuizen Nucl. Phys. B389, (1993), 25

[19] A.M.Polyakov, in Field, Strings and Critical Phenomena, (1989), ed. E.Brezin, J.Zinn-Justin, Les Houches

[20] V.G.Knizhnik, A.B.Zamolodchikov Nucl. Phys. B247, (1984), 83 P.Fre, F.Gliozzi Nucl. Phys. B326, (1989), 411

[21] A.A. Belavin, A.M.Polyakov, A.B.Zamolodchikov Nucl. Phys. B241, (1984), 333

[22] Z.Horvath, L.Palla, P.Vecsernyes Int. J. Mod. Phys. A4, (1989), 5261;

[23] T.Kuramoto Nucl. Phys. B346, (1990), 527

[24] E.D' Hocker, R.Jackiw Phys. Rev. D26, (1982), 3517

[25] J.Kim, T.Lee Phys. Rev. D42, (1990), 26

[26] R.C.Myers, V.Periwal, Nucl. Phys. B397, (1993), 239

[27] J.Distler, Z.Hlousek, H.Kawai Int. J. Mod. Phys. A5, (1990), 391 
[28] Y.Oz, J.J.Pawelczyk, S.Yankielowicz Nucl. Phys. Lett. B363, (1991), 555

[29] I.R.Klebanov Phys. Rev. D51, (1995), 1836

[30] I.R.Klebanov, A. Hashimoto Nucl. Phys. B434, (1995), 264 